\title{
Placebo: Análisis conceptual de un constructo en transición*
}

\section{Joseph W. Critelli \\ Karl F. Neumann}

Nortb Texas State University

Traducido por M.`A. Ruiz y M. S. Blanco

En algunas revisiones recientes se ha cuestionado la lógica del constructo placebo (Kirsch, 1978) y la necesidad de emplear grupos de control placebo en la investigación de la eficacia de la psicoterapia (O'Leary y Borkovec, 1978; Wilkins, 1979a, 1979b, 1983). Nosotros creemos que las opiniones vertidas en estos artículos son respuestas a la evolución actual hacia el empleo de conceptualizaciones más cognitivas en la psicoterapia. En efecto, esta transición ha afectado al lenguaje utilizado en la caracterización de los efectos del placebo: un reciente intercambio de ideas entre destacados investigadores en este área (Bootzin y Lick, 1979; Kazdin, 1979a, 1979b) terminó con la conclusión de que nuestro «sistema conceptual actual no ha conseguido funcionar como vehículo adecuado a través del cual puedan discutirse y resolverse problemas empíricos y teóricos» (Wilkins, 1979b, pág. 859). Este artículo explora posibles soluciones al problema del placebo en este período de transición.

\section{BREVE HISTORIA}

La noción de placebo nació en medicina para explicar la sorprendente efectividad de procedimientos novedosos, especialmente cuando los administraban defensores entusiastas de su eficacia (Frank, 1961; Shapiro, 1971). De hecho, Shapiro describe la historia de la medicina hasta 1600 como la historia del efecto placebo. Aun cuando ello haya supuesto la utilización de técnicas con frecuencia realmente perjudiciales para la salud, todas las sociedades, desde los albores de la humanidad, han aceptado el poder terapéutico de tratamientos sancionados por sus comunidades (Frank, 1961). Finalmente, llegó un momento en que probar todo fármaco nuevo comparándolo con placebos farmacológicos inertes se convirtió en práctica común en la medicina. La mejoría del cliente no se consideraba, por sí sola, apoyo suficiente de la acción específica de un remedio (ver Shapiro, 1971) o de la teoría de curación que la subyacía. No es que los placebos fueran ineficaces; por definición, cualquier placebo tenía al menos eficacia suficiente como para mantener su aceptación dentro de la comunidad en la que había surgido. Más aún, a los placebos se les ha atribuido curaciones verdaderamente sorprendentes (Byerly, 1976; Frank, 1961). Pero los placebos no producían curación en todos los casos, y

* Enero 1984. American Psychologist, vol. 39, 1, págs. 32-39. 
muchos creían que no podían mejorar para producir efecros terapéuticos mayores y más consistentes. Así, con la llegada de drogas y técnicas quirúrgicas que mostraban efectos superiores a los del placebo, la etiqueta de placebo adquirió una connotación negativa en medicina.

Rosenthal y Frank (1956) fueron en gran medida los responsables de la extensión del constructo placebo a los tratamientos psicológicos. Ellos sugirieron que la psicoterapia podía compararse con un tratamiento placebo que fuera considerado terapéuticamente inerte desde el punto de vista de la teoría de la terapia que estaba siendo evaluada. Como en la investigación con drogas, la mejoría terapéutica no era el aspecto clave; la mejoría del cliente, por sí sola, se consideraba insuficiente para apoyar la actividad específica de las técnicas aplicadas, o la teoría desde la que fueron generadas. Se consideraba que cualquier procedimiento con credibilidad, y aplicado de una forma socialmente aceptable, produciría probablemente una cierta mejoría, y ésta era precisamente la razón por la que debían controlarse estos efectos placebo.

Al menos desde los tiempos de Watson podríamos decir que la psicología y la psicoterapia se han situado en el mismo continuum que las ciencias físicas y el tratamiento médico. Por tanto, era muy natural que la psicología extendiera a partir de la medicina el uso del control placebo y adoptara la actitud de la medicina hacia los efectos placebo. De esta forma, el placebo se consideró como una variable «molesta» (Bernstein y Neitzel, 1977), algo que no era de interés en sí mismo, pero que había que controlar a fin de validar los tratamientos terapéuticos reales.

Los placebos no sólo se consideraban menos eficaces que los tratamientos en sí, sino que de algún modo también se veían como menos reales. Los tratamientos operaban mediante «ingredientes activos», a diferencia de sus correspondientes placebos, supuestamente inertes. Además, los efectos placebo, mediatizados por las expectativas, no encajaban en la visión del mundo conductista donde las cogniciones se consideraban una ficción, y el pensamiento quedaba fuera de las cadenas causales que vinculaban los acontecimientos dentro del mundo real (Skinner, 1974). A pesar de la falta de demostración de que los placebos fueran menos potentes que las terapias establecidas (Frank, 1961; Kazdin, 1979b; Rosenthal y Frank, 1956), en general fueron objeto de menosprecio considerándose sus efectos como «artefactuales».

Con el surgimiento de los modelos cognitivo-conductuales se produjo, sin embargo, un cambio sutil en la formulación del objeto de la psicología. Con este cambio, empezó a caerse en la cuenta de que, como variable cognitiva, el placebo podría ser de interés en sí mismo (Kirsch, 1978). Pero esta idea también trajo con ella la sospecha de que los efectos de las terapias cognitivas, y quizás también de cualquier otra forma de psicoterapia, podrían no tener más efectos que los de los placebos. (Bootzin y Lick, 1979; Kazdin, 1979a; Kirsch, 1978). Estos cambios en los supuestos subyacentes a la naturaleza del cambio terapéutico han sido responsables de matizaciones en la terminología con la que se describen los efectos placebo, y también han sido el origen de presiones, bien en el sentido de prescindir totalmente del constructo placebo o en el de reinterpretarlo de forma que refleje la aceptación general de formulaciones más cognitivas (Kirsch, 1978).

\section{LA TERMINOLOGIA: ASPECTOS DENOTATIVOS}

Dos son las metáforas dominantes que pueden encontrarse en la literatura con respecto al placebo: (a) los placebos como ingredientes inertes en vez de activos, y (b) los placebos como agentes no específicos en vez de especí- 
ficos. Aunque particularmente problemática en los últimos años, es importante señalar que la terminología del placebo en ambas metáforas ha constituido siempre una especie de tela de araña de sobregeneralizaciones y contradicciones internas. Nosotros revisaremos aquí las distintas estrategias para definir el placebo en psicología y trataremos de indicar cuáles de estas estrategias parecen actualmente más viables.

\section{Placebo como tratamiento inerte}

El método de investigación utilizado en medicina en el que se comparaba un agente químico activo con una sustancia farmacológicamente inerte tiene bastante sentido. Incluso aunque estas sustancias inertes fueran activas en el sentido de producir efectos observables, no eran farmacológicamente activas. Así, las comparaciones droga-placebo permitían hacer atribuciones con respecto a la acción química específica o a la interacción entre factores químicos y psicológicos, además de los factores psicológicos no específicos compartidos por la droga y el placebo.

$\mathrm{Al}$ aplicar el modelo médico a la psicoterapia, se mantuvo la metáfora del tratamiento activo versus placebo inerte. Este modelo, sin embargo, nunca se ajustó muy bien al campo de la psicoterapia. En realidad, tanto el tratamiento experimental como el control placebo eran, por supuesto, psicológicamente activos y farmacológicamente inertes.

Como ya hemos señalado, Rosenthal y Frank (1956) aplicaron la metáfora activo-inerte al estudio de la psicoterapia, revisando la definición de placebo y convirtiendo el «farmacológicamente inerte» en "terapéuticamente inerte desde el punto de vista de la teoría de la terapia en estudio» (pág. 299). Hay que hacer aquí una distinción fundamental entre lo teóricamente inerte y terapéuticamente inerte. Con demasiada frecuencia, la definición de Rosenthal y Frank ha sido malinterpretada en el sentido de que los placebos son terapéuticamente inertes, esto es, no tienen efectos terapéuticos. Por ejemplo, O'Leary y Borkovec (1978) afirman que los placebos son teóricamente inertes y por consiguiente no son «tratamientos potencialmente efectivos» (pág. 824). No hay nada en la historia de los efectos placebo en medicina o en psicología que apoye la creencia de que los placebos son terapéuticamente inertes. Por tanto, que una teoría dada prediga o no un efecto terapéutico debe considerarse como un problema distinto del de la eficacia real.

El problema fundamental que tiene la definición de Rosenthal y Frank es que prácticamente todas las formas de psicoterapia actuales podrían considerarse inertes, y por tanto placebo, desde el punto de vista de otras teorías de curación. La mayoría de los psicólogos preferirían probablemente un poco más de estabilidad en aquello a lo que el placebo se refiere. Una solución posible es utilizar la definición de O'Leary y Borkovec (1978), según la cual podría considerarse placebo a aquella condición con respecto a la que «actualmente no se dispone de razón teórica por la que... tendría que esperarse que debiera influir sobre la conducta en cuestión» (pág. 823). Bandura (1977), sin embargo, ha desarrollado recientemente la teoría de la autoeficacia, que afirma que «cambios logrados por métodos diferentes [de terapia] son atribuibles a un mecanismo cognitivo común» (pág. 191). Bandura mantiene que «los procedimientos psicológicos, cualquiera que sea su forma, alteran el nivel y fuerza de la autoeficacia» (pág. 191). El postula que estos cambios en la autoeficacia afectan a la iniciación y persistencia de conductas de enfrentamiento, y por consiguiente, la autoeficacia puede utilizarse para explicar y predecir los cambios logrados por diferentes tipos de tratamientos. Por ello, Ban- 
dura ha proporcionado una razón teórica con apoyo reciente para predecir que cualquier procedimiento que altere las expectativas de eficacia personal debe ser terapéuticamente efectivo. Puesto que la autoeficacia puede recibir la influencia de diversos procedimientos, tales como conductas directas, experiencia vicaria, persuasión verbal y activación fisiológica, parece probable que todos los tratamientos placebo conocidos, además de sus otros efectos, podrían afectar a la autoeficacia. Por tanto, la llegada del modelo de autoeficacia crea problemas a la redefinición de O'Leary y Brokovec. Bajo esa redefinición, ningún tratamiento que afecte a la autoeficacia podría considerarse como placebo. En efecto, podría descartarse la existencia del placebo. Aunque esta idea podría parecer razonable a primera vista, hay que señalar que esto no solucionaría los problemas fundamentales que provocó la extensión del constructo placebo a la psicoterapia.

Un repaso a la historia de las profesiones relacionadas con la terapia (Frank, 1961; Shapiro, 1971; Shapiro y Morris, 1978) revela que, por lo general, de prácticamente cualquier tratamiento con credibilidad, sancionado socialmente y administrado correctamente, puede esperarse un efecto terapéutico moderado. Por consiguiente, se necesitan métodos que aseguren que tiene un fundamento real la pretensión inevitable de las terapias nuevas de tener una «eficacia superior» o de que sus ingredientes activos tengan «actividad específica». Como mostraremos, tales métodos dependen directa o indirectamente del constructo placebo.

\section{El placebo como tratamiento no específico}

Antes de examinar la metáfora de la inespecificidad deberíamos aludir a la distinción entre factores placebo independientes y efectos placebo dependientes. Los factores placebo incluyen variables independientes relacionadas con el cliente, terapeuta, contexto y técnica, así como sus interacciones mutuas. Los efectos placebo se refieren a los efectos dependientes de estos fac: tores, entre los que se incluyen cambios en las expectativas, sentimientos, cogniciones, respuestas fisiológicas y conductas manifiestas del cliente. Variables mediacionales, tales como expectativas de curación, pueden operar como factores o como efectos, dependiendo del contexto y técnica, así como sus interacciones mutuas. Los efectos placebo se refieren a los efectos dependientes de estos factores, entre los que se incluyen cambios en las expectativas, sentimientos, cogniciones, respuestas fisiológicas y conductas manifiestas del cliente. Variables mediacionales, tales como expectativas de curación, pueden operar como factores o como efectos, dependiendo del contexto y de las atribuciones de causalidad de la persona. El término «efectos no específicos»se utiliza a menudo en la literatura para indicar tanto efectos dependientes como manipulaciones independientes. Un ejemplo de ello es la siguiente afirmación de Kazdin y Wilcoxon (1976) de «que los efectos terapéuticos son debidos a efectos no específicos del tratamiento» (pág. 745). Cuando se relacionan causalmente dos conjuntos de eventos, la terminología empleada debería permitir distinguir entre lo que se considera causas y lo que se considera efectos.

La reciente proliferación de significados de la denominación «no específico» explica una parte importante de la confusión dentro de la literatura sobre el placebo. Se han empleado tres versiones principales de la metáfora de la inespecificidad. En general, estas interpretaciones han sido utilizadas intercambiablemente en la literatura, sin reconocer sus diferentes implicaciones en la determinación de qué tratamientos podrían clasificarse como placebos y cuáles no. 
Sin actividad específica. En la primera definición, Shapiro (1971) define el placebo como «un procedimiento sin actividad específica con respecto a la condición que se está evaluando» (pág. 440). Esta definición se deriva de la medicina y responde a la lógica de que un remedio que se ofrece como panacea de solución de muchos problemas diferentes es improbable que opere a través de una única acción química. Esta definición tiene cierto sentido dentro de la medicina, pero muy poco cuando se aplica a la psicoterapia. Se basa en la suposición de que no existe ningún mediador central, con base fisiológica, de la salud y de la autocuración. Si existiese ese mediador, a los remedios que estimularan este mecanismo autocurativo podría atribuírseles legítimamente una acción específica responsable de efectos curativos generales sobre una serie de problemas diferentes. Este supuesto puede ser plausible dentro de la medicina, pero la psicología ofrece distintos sistemas teóricos reconocidos en los que se proponen precisamente esos mediadores centrales de la mejoría terapéutica (es decir, la tendencia a la actualización de Rogers, la autoeficacia de Bandura). Por tanto, el criterio de la actividad específica es bastante inapropiado para tratar el placebo en psicoterapia.

Características no especificadas. Un segundo uso del término «inespecífico» se refiere a aquellas características del tratamiento que no se han especificado como los «ingredientes activos» de una psicoterapia concreta (Jacobson y Baucom, 1977; Mahoney, 1977; Shapiro y Morris, 1978). La denominación «no especificados» parecería más apropiada que la tradicional «inespecíficos». El problema que surge con este criterio es el de la ambigüedad con respecto al grado de especificación necesario para que un procedimiento deje de llamarse placebo. ¿Sería suficiente especificación darle nombre a una variable, o sería necesario también un consenso con respecto a cómo influye ese mecanismo, al que hemos puesto nombre, sobre la mejoría (Kirsch, 1978)? La expectativa de mejora, por ejemplo, se ha considerado tradicionalmente como una variable central del placebo. ¿Constituiría la teoría de la autoeficacia una especificación suficiente como para que dejaran de definirse como placebos todos aquellos tratamientos basados en las expectativas de mejora? Como vemos, dificultades como éstas a la hora de aplicar este criterio hacen su adopción problemática.

Factores comunes. La tercera gran interpretación del término «inespecífico" se refiere a los factores placebo que no son específicos a tratamientos concretos, es decir, factores que son compartidos por la mayoría de las terapias (Frank, 1961; Kazdin, 1979a; Wilkins, 1979a). De acuerdo con Kazdin (1979a), podría mantenerse el término «inespecíficos» o bien denominarse «factores comunes». Parecería que la especificación de los factores que todas las terapias tienen en común tiene un carácter menos interpretativo que la identificación de sus ingredientes activos, de modo que la definición basada en los factores comunes gozaría de una objetividad relativa mayor. Los factores comunes de la psicoterapia coinciden básicamente con las listas tradicionales de variables placebo, es decir, factores tales como sugestión, persuasión, credibilidad del tratamiento, atención del terapeuta, expectativas de curación, y demanda de mejoría. Así pues, el criterio de los factores comunes es consistente desde un punto de vista lógico, no es difícil de aplicar, es relativamente objetivo y consonante con las variables tradicionales del placebo. Como tal nos parece la definición actualmente más viable del placebo en el estudio de la psicoterapia. 
Kirsch (1978) cree que el problema del placebo, a la luz del auge actual que viven los modelos cognitivos, es que ciertas técnicas cognitivo-conductuales que gozan de aceptación podrían clasificarse como placebos y por consiguiente ser objeto de rechazo. Basándose en esta idea, concluye que el placebo no puede definirse con fundamento dentro de la psicología. En el análisis de Kirsch parece confundirse el aspecto denotativo, definicional del placebo, con su connotación negativa («sólo es un placebo»). Una vez que se hace esta distinción se puede ver, como ya hemos argumentado, que el placebo puede definirse adecuadamente en la psicología desde un punto de vista denotativo. Permanecen, sin embargo, los problemas relacionados con la connotación negativa del placebo, que debemos analizar con más detenimiento.

Esta connotación negativa parece que procede de cuatro fuentes. En primer lugar recordemos que la noción del placebo (algo que debe controlarse pero no estudiarse por derecho propio) surgió en medicina, donde la acción farmacológica, y no la psicológica, era el objeto de estudio. Este argumento histórico carece de fundamento en psicología.

La segunda fuente de la connotación negativa del placebo procede del supuesto mantenido implícitamente de que sólo hay un nivel de «efecto placebo» y que, consecuentemente, no pueden desarrollarse tratamientos que induzcan más mejoría que un "placebo estándar». Este punto de vista es el resultado de una malinterpretación del criterio de los factores comunes. Por ejemplo, aunque la sugestión aparece en una amplia gama de terapias, sería inadecuado suponer que adopta la misma forma o intensidad en todas ellas, o que interactúa con otros componentes terapéuticos exactamente en la misma forma en todas las terapias. Así, pues, es importante señalar que el criterio de los factores comunes no dice que todos los factores comunes deben mostrar el mismo nivel de eficacia. En artículos recientes ha empezado a desvanecerse esta variante del supuesto de uniformidad, y se está comenzando ahora a investigar seriamente la posibilidad de desarrollar tratamientos más eficaces basándose en una mejor comprensión de los factores relacionados con el placebo (Bandura, 1977, 1982; Bootzin y Lick, 1979).

Un tercer argumento en contra del placebo se basa en la idea de que el interés por los efectos del placebo «interfiere» con el estudio de los mecanismos «reales» de curación. Este argumento, que se debe a una comprensión inadecuada de la metáfora del placebo como «inerte», ignora totalmente el hecho indiscutible de que los placebos poseen una eficacia terapéutica real y empíricamente demostrable. Así pues, además de otros ingredientes potencialmente activos, hay que comprender y utilizar más adecuadamente los mecanismos del placebo.

La cuarta fuente de connotaciones negativas del placebo no puede rechazarse tan alegremente. Habría que recordar que todas las interpretaciones actuales del placebo lo definen como una categoría «cajón de sastre» de una amplia variedad de variables (Kazdin, 1979a). Artefactos de medida tales como los efectos de la práctica, por ejemplo, se han considerado tradicionalmente como factores placebo (Shapiro y Morris, 1978). Por tanto, en la medida en que estos aspectos extraños al tratamiento inflen los resultados terapéuticos, la connotación negativa del placebo podría ser en parte merecida.

Para ayudar a asegurar que las terapias actuales no cargan inapropiada o indiscriminadamente con esta connotación negativa, parece fundamental separar terminológicamente los artefactos de medida de los factores placebo reales, psicológicamente activos. Hay que decir, además, que según la definición basada en los factores comunes, los artefactos de medida no pueden calificar- 
se como variables placebo. Una vez que se ve claramente que los factores placebo no tienen efectos terapéuticos reales, parece obvio que la discriminación crítica en la evaluación de la eficacia de una terapia no está entre el placebo y el tratamiento «real», sino entre resultados genuinos y resultados debidos a artefactos. Nosotros sugerimos, por consiguiente, que debe distinguirse entre los verdaderos efectos placebo y los artefactos de medida. Con esta distinción puede verse que la connotación negativa del placebo es inmerecida.

\section{Utilidad del constructo placebo en la evaluación de la eficacia de la psicoterapia}

El término «eficacia» se utiliza en la literatura sobre el placebo al menos de dos formas. La primera hace referencia al hecho de si el tratamiento facilita la mejoría del cliente, y en su aplicación práctica, esta eficacia de línea base es la que constituye el fundamento para la recomendación del tratamiento. La segunda forma, la eficacia incremental, se refiere a si el tratamiento ha demostrado un nivel de eficacia superior a lo que se esperaría de cualquier procedimiento con una alta credibilidad y administrado de una forma aceptada socialmente. La eficacia incremental no tiene relación directa con la prescripción de tratamiento. Desde un punto de vista pragmático debería recomendarse el mejor tratamiento de que se disponga, independientemente de que opere a través de mecanismos placebo.

La eficacia de línea base se juzga normalmente por índices de curación, diferencias pre-post y por comparaciones entre controles de lista de espera y no-tratamiento. Wilkins (1979a) ha mostrado también que controles placebo de baja credibilidad también son relevantes a la eficacia línea base, puesto que permiten atribuir el cambio a los propios procedimientos terapéuticos más que a variables situacionales. Los resultados positivos en términos de eficacia de línea base indican simplemente que algo relacionado con el tratamiento ha actuado de forma tal que ha conseguido producir una mejoría en el cliente a partir de sus experiencias normales por sí solas. $Y$ aunque la eficacia de línea base debe demostrarse independientemente de la actuación de artefactos de medida, nosotros no vemos tales artefactos como variables placebo.

La eficacia incremental, a diferencia de la de línea base, proporciona una perspectiva histórica y transcultural con arreglo a la que se puede comparar la magnitud de los efectos actuales de un procedimiento con los de otros tratamientos surgidos en distintas sociedades humanas a lo largo de la historia. Funciona como punto de referencia para determinar dónde se sitúan las terapias actuales dentro de ese marco general. Podría argumentarse que el énfasis puesto sobre la eficacia incremental en psicología se deriva de una adopción acrítica de los métodos de la medicina. Los diseños empleados para validar los efectos farmacológicos de las drogas pueden ser sencillamente inapropiados para evaluar la eficacia de la psicoterapia. Podría darse el caso, por ejemplo, de que los factores placebo englobaran legítimamente todos aquellos ingredientes que son activos en psicoterapia. Si esto fuera cierto, plantear el tema de la eficacia incremental podría equivaler al ejercicio autoengañoso de preguntarse si los tratamientos psicológicos tienen efectos más allá de los propiamente psicológicos. Por otro lado, se ha defendido la eficacia incremental de muchas formas de psicoterapia, y en la medida en que sigue haciéndose esta defensa, debe someterse a evaluación.

Disponemos en principio de cuatro estrategias básicas para establecer la eficacia incremental. La primera implica la comparación entre la efectividad de un tratamiento dado y la evidencia a favor de la eficacia de otras terapias 
recogida en revisiones meta-analíticas, cuando se tienen en cuenta variables moduladoras como gravedad del síntoma, duración del tratamiento y forma de evaluación de los resultados terapéuticos (Smith y Glass, 1977). Este método es particularmente deseable en términos coste-beneficio puesto que no requiere el uso más costoso de otros grupos de control distintos de los tradicionales de no-tratamiento. Sin embargo, se supone, cuando se utiliza, que otros tratamientos existentes son relativamente uniformes en cuanto a su eficacia. De otra forma, no estaría claro qué niveles de mejoría servirían de criterio para establecer la eficacia incremental. En este momento, este supuesto parece viable (Smith y Glass, 1977). Un problema más serio es la tendencia que parecen mostrar terapias nuevas a ser más eficaces que otros tratamientos de uso habitual (Frank, 1961; Shapiro, 1971). Puesto que las revisiones meta-analíticas de las investigaciones realizadas incluyen muchas terapias viejas, una eficacia mayor que la del promedio no se podría tomar como una demostración clara de efectos incrementales. Quizá puede aplicarse la flexibilidad del meta-análisis para paliar parcialmente al menos este problema, por ejemplo comparando el tratamiento en prueba con otras terapias investigadas cuando eran nuevas.

El uso del meta-análisis para conseguir evidencia de que existe eficacia incremental parece prometedor, aunque con frecuencia esta evidencia no sea demasiado concluyente. Debido a su bajo coste, sin embargo, el meta-análisis podría usarse sistemáticamente para disponer de evidencia inicial de que un tratamiento puede haber logrado efectos incrementales. Hay que señalar que incluso aunque el método meta-analítico no emplea un grupo de control placebo, la selección de variables moduladoras y de los criterios de inclusión de tratamiento se basa implícitamente en la conceptualización de los efectos placebo.

El segundo procedimiento para encontrar apoyo a la eficacia incremental consiste en comparar el tratamiento en estudio con una terapia establecida (O'Leary y Borkovec, 1978; Wilkins, 1979a). Se supone que la historia de las pruebas de que un tratamiento supere a otras terapias reconocidas constituiría evidencia de eficacia incremental. Una limitación de esta estrategia radica en que la elección de terapias existentes está relativamente determinada, de modo que el tratamiento en estudio y la terapia establecida no podrían emparejarse normalmente en variables relevantes relacionadas con el estilo y el procedimiento, como sugieren Jacobson y Baucom (1977). Puesto que este emparejamiento es esencial para determinar qué mecanismos teóricos y componentes del tratamiento explican el cambio, las comparaciones con una terapia establecida no resulta ser una estrategia de investigación útil. Por otro lado, el no encontrar diferencias entre el tratamiento en estudio y la terapia establecida puede considerarse fácilmente como un éxito del tratamiento de cara a su eficacia incremental, y no como fracaso. Esta podría ser una forma de evitar la connotación negativa un tanto crónica del placebo. Hay que reconocer, sin embargo, que en este método la terapia establecida está utilizándose como grupo de control placebo, y por lo tanto se debe demostrar que ambos tratamientos, el que se está estudiando y la terapia establecida, no difieren en las variables placebo tradicionales, como la credibilidad, la atención del terapeuta, etc.

Un tercer método para demostrar la eficacia incremental podría consistir en la comparación del tratamiento en prueba con uno o más de sus grupos de control componentes. Como con el método de la terapia establecida, quedará todavía por demostrar que el tratamiento completo no difiere de sus controles componentes en cada una de las variables placebo relevantes. Esta estrategia tiende a ser más eficaz que el control de la terapia establecida, puesto 
que aporta información sobre los mecanismos y componentes del cambio al mismo tiempo que una comparación relevante a la determinación de la eficacia incremental. Un inconveniente es, sin embargo, que un tratamiento inferior desde un punto de vista global, podría superar teóricamente a sus propios controles componentes sin establecer necesariamente su verdadera eficacia incremental. Habría que comparar entonces los resultados de este método con los conseguidos mediante otros procedimientos para demostrar la eficacia incremental.

El cuarto método consiste en hacer una comparación entre el tratamiento en estudio con el grupo de control placebo tradicional (es decir, un tratamiento ad hoc cuya eficacia se cree que se deriva únicamente de factores comunes). Un problema que presenta esta estrategia es la dificultad de mantener al terapeuta realmente ignorante de su asignación a la condición experimental o de control. Esta dificultad imposibilita la realización de estudios de verdadero doble-ciego. La estrategia del grupo de control placebo se ha criticado a menudo por razones éticas (v., O'Leary y Borkovec, 1978; Wilkins, 1979a) que se supone que no se tienen en cuenta cuando se asigna a clientes a un tratamiento ineficaz. El origen de esta crítica está en una comprensión inadecuada del problema. Hemos de repetir que aunque los placebos se han considerado ineficaces desde el punto de vista teórico, sí son efectivos terapéuticamente. $\mathrm{Y}$ aunque los grupos de control placebo han solido ser en el pasado menos eficaces empíricamente que los tratamientos establecidos, es razonable atribuir esto a una proclividad no intencionada a diseñar placebos débiles, más que a limitaciones inherentes a la eficacia del tratamiento placebo. En resumen, hay que reconocer que el procedimiento del grupo de control placebo proporciona la respuesta más directa y clara a la pregunta de si el nivel de eficacia que muestra un tratamiento es superior al de los efectos de su placebo. Desde luego, igual que en el método de la terapia establecida, hay que tener cuidado de asegurarse y demostrar que los grupos no difieren en las variables placebo principales.

Dada la tendencia universal en todas las sociedades a considerar sus propias formas de «sanar» al uso como más eficaces y activas que los rituales utilizados en otros momentos del pasado, nosotros sugerimos que para abordar el problema de la eficacia incremental es necesario emplear los cuatro métodos que hemos descrito. En conclusión, podemos decir que, aunque el constructo placebo no sirva de mucho a la hora de determinar la eficacia línea base, sí es bastante necesario para evaluar los efectos incrementales.

\section{¿Son las terapias actuales más eficaces que el tratamiento inespecífico?}

El requisito tradicional para demostrar la eficacia de la psicoterapia ha sido mostrar que es más eficaz que el tratamiento inespecífico (Eysenck, 1961; Kazdin, 1979a; Kazdin y Wilcoxon, 1976; Paul, 1966; Shapiro, 1971). Wilkins (1979a, 1979b), O'Leary y Borkovec (1978) y con toda probabilidad la gran mayoría de los psicoterapeutas han afirmado que este criterio se ha conseguido.

Frank (1961) y Shapiro (1971) han defendido que, puesto que todas las culturas tienen formas de terapia que son aceptadas como válidas dentro de sus sociedades, y puesto que todas las terapias muestran aproximadamente el mismo nivel de eficacia, es probable que las semejanzas entre estos distintos procedimientos sean más importantes que sus particularidades. De esta forma, los agentes curativos en estas terapias podrían ser factores inespecíficos más que los supuestos «ingredientes activos» a los que se ha atribuido el cam- 
bio tradicionalmente. Aunque las revisiones realizadas por Frank y Shapiro no son nuevas y sus argumentos no se han refutado, el campo en su conjunto ha seguido asumiendo que la mayor parte de los efectos terapéuticos es atribuible a componentes y mecanismos especificados del tratamiento, y que las terapias actualmente populares son superiores al tratamiento inespecífico (Wilkins, 1979a).

El problema está, sin embargo, en qué es lo que debe demostrarse para reconocer la existencia de eficacia terapéutica. Las primeras investigaciones sobre psicoterapia, estrechamente relacionadas con la utilización médica de los controles placebo en la farmacología, contemplaban los efectos del placebo como «irreales» o artefactuales. Así pues, se consideraba necesario para demostrar la eficacia terapéutica encontrar efectos superiores a los que se producían en condiciones placebo. Sin embargo, como ya hemos defendido, no hay nada de irreal en los efectos terapéuticos de los placebos mientras estos efectos se distingan de los artefactos de medida. Por consiguiente, la eficacia terapéutica puede establecerse en función de los efectos sobre la línea base, más que de los incrementales. Parece haber poca duda en que la mayoría de las terapias establecidas han demostrado un nivel de eficacia de línea base.

No obstante, a la luz de investigaciones recientes, va haciéndose cada vez más difícil mantener la fe en la eficacia incremental de las terapias existentes. Luborsky, Singer y Luborsky (1975) revisaron la investigación «bien controlada» sobre resultados terapéuticos y concluyeron que todas las terapias existentes poseen una eficacia similar. Garfield (1981) también ha revisado recientemente la historia y estado actual de la psicoterapia y ha concluido que, a pesar de la gran diversidad de psicoterapias existentes, no se han producido avances significativos.

Smith y Glass (1977) no encontraron diferencias en su revisión metaanalítica entre procedimientos de orientación conductual y no conductual. Estos autores calificaron de «modestos» los efectos terapéuticos promedio a pesar de la actuación de sesgos o artefactos favorables a los efectos de los tratamientos. Por ejemplo, tanto clientes como terapeutas emplearon una buena parte de su tiempo y de su esfuerzo en el objetivo de «curarse». Podríamos pensar que para justificar este esfuerzo, los clientes experimentaron una cierta demanda de presentarse como «mejorados» en la evaluación post-tratamiento. Los clientes-control asignados a la lista de espera que aún se presentaban para ser curados, podrían haber experimentado una demanda opuesta. Así, pues, parece razonable suponer que una cierta parte, probablemente limitada, de estos efectos «modestos» deben atribuirse a artefactos de demanda. Smith y Glass encontraron también que ni la duración del tratamiento ni la experiencia del terapeuta correlacionaba con la magnitud de la eficacia terapéutica. Sus resultados constituyen un serio apoyo a los análisis de Frank (1961) y Shapiro (1971).

No es sorprendente que en la evaluación de la eficacia de las terapias actuales frente al tratamiento inespecífico se hayan hecho fundamentales los problemas de terminología. La historia de la investigación en psicoterapia nos sugiere que tampoco es sorprendente el hecho de que no hayan faltado lanzas a favor de la superioridad general (es decir, eficacia incremental) de las terapias populares. Wilkins (1979a, pág. 838), por ejemplo, sostiene que la desensibilización sistemática es un procedimiento «más potente» que las terapias de los años cincuenta y que sus efectos son superiores a los de los procedimientos placebo (Wilkins, 1979b, pág. 859). Basa esta conclusión en la capacidad de la desensibilización sistemática para superar a los controles de baja, aunque no de alta, credibilidad.

Aunque las primeras investigaciones sobre resultados terapéuticos incluían 
normalmente grupos de control inespecifico que no tenian una credibilidad alta, en su momentto se tomaron como controles adecuados de los efectos inespecíficos. Recientemente, y en especial desde la revisión de Kazdin y Wilcoxon (1976), para considerar adecuado un procedimiento de control se le ha exigido que demostrara un grado igual de credibilidad y expectativas de curación. Es probable también que los criterios de adecuación de los controles placebo se hagan más exigentes en el futuro. Posiblemente se les exija la demostración de equivalencia en otras variables placebo tales como sugestión, demanda de mejoría, etc. Es importante reconocer que, tal y como están las cosas, el que el tratamiento supere a un control placebo de baja credibilidad de ninguna manera demuestra la existencia de eficacia incremental. Aunque la mayoría de las terapias actuales han demostrado eficacia línea base, si se pueden desarollar fácilmente procedimientos placebo igualmente eficaces y creíbles, como parece ser el caso (Lick, 1975; McReynolds, Barnes, Brooks y Rehagen, 1973; Neumann, Critelli y Schneider, 1981; Tori y Worrell, 1973), sería erróneo tomar esa eficacia como una prueba de la «superioridad» general de una terapia (Wilkins, 1979a, pág. 840).

\section{CONCLUSION}

¿Podríamos inferir de todo lo anterior que las terapias actuales no tienen por qué ser más efectivas que la fe en la curación o los rituales de las sociedades primirivas? En la medida en que la magnitud y la fiabilidad de los efectos del placebo son uniformes en distintos momentos y culturas, la respuesta podría ser sí. $Y$ dado que no hay datos claros que demuestren lo contrario, ese sí sería consecuente con una actitud científica conservadora. Sin embargo, las comparaciones directas entre nuestras terapias actuales y las de otras culturas pueden no tener sentido, dado que la credibilidad de un tratamiento es específica a su cultura. Además, desde un punto de vista histórico vemos cómo lo que es «científico» dentro de una cultura puede caer en lo «mágico» o «supersticioso» en otras. Lo que es diferente y verdaderamente más científico en las terapias actuales es la precisión con la que se las somete a prueba, se las revisa, perfecciona, o se las descarta. Esta primacía de la evaluación empírica mediante metodologías de comparación controladas, parece probable que dé como resultado un desarrollo gradual de procedimientos cada vez más eficaces al margen de que operen o no por mecanismos placebo tradicionales.

Pero si queremos garantizar este progreso, es indispensable que los tratamientos en prueba se comparen con controles placebo realistas. La atribución injustificada a un tratamiento de eficacia incremental se ha debido en el pasado, con mucha frecuencia, al uso experimental de placebos que ni la persona más ingenua podría confundir con una terapia auténtica. Parece haber una tendencia a emplear placebos experimentales en cierto sentido débiles, menos creíbles, o aplicados con menos entusiasmo que los tratamientos considerados como terapias reales. Como mínimo, los controles placebo deberían ser equivalentes a los procedimientos en prueba en todos los factores comunes reconocidos más importantes. Entre éstos se podrían incluir las expectativas inducidas de mejoría, la credibilidad de la argumentación teórica, la credibilidad de los procedimientos, la demanda de mejoría; y por parte del terapeuta, su atención, entusiasmo, esfuerzo, creencia percibida en los procedimientos terapéuticos, y compromiso con la mejoría del cliente.

Es importante señalar, sin embargo, que la equivalencia relativa entre las terapias actuales y el tratamiento inespecífico de ninguna forma impugna o minimiza la eficacia terapéutica de estos procedimientos. Los procedimientos 
psicoterapéuticos actuales son herramientas de curación legítimas y las naturales en nuestra sociedad, y como tales juegan un papel crucial y quizá indispensable en el mantenimiento del equilibrio entre las necesidades individuales y las sociales. La perdurabilidad de las prácticas curativas persona-a-persona en todas las sociedades humanas conocidas atestigua la necesidad de una institución social a través de la cual se pueda paliar el sufrimiento individual, las dificultades interpersonales y las desviaciones problemáticas. De hecho, puede decirse que la psicoterapia actual y sus predecesores a través de todas las épocas representan un componente inherente de la cultura humana. En cualquier lugar, momento o circunstancia bajo las que surja una sociedad se desarrollarán espontáneamente prácticas terapéuticas. En ese contexto, la necesidad e importancia de la psicoterapia sigue siendo incuestionable.

También es cierto, sin embargo, que las terapias actuales aún no han respondido al reto de demostrar efectos incrementales. Así pues, las especificaciones que se están haciendo sobre sus mecanismos y componentes activos deben considerarse tentativas. Estos tratamientos pueden estar funcionando a través de sus factores comunes. También puede que estén actuando como factores especificados, y la suposición de que estos mecanismos especificados son más potentes que los factores placebo puede ser errónea. En cualquier caso, hay que reconocer que la reputación científica de las terapias actuales se fundamenta en los métodos precisos mediante los que se evalúan y perfeccionan. Esto es lo que las diferencia de las prácticas curativas del pasado. $\mathrm{Y}$, puesto que el placebo ha de jugar un papel crucial en la evaluación científica de la psicoterapia, pensamos que rechazarlo prematuramente constituiría una regresión conceptual y metodológica.

\section{Referencias}

BANDURA, A.: «Self-efficacy: Toward a unifying theory of behavioral change». Psychological Review, 1977, 84, 191-215.

Bandura, A.: «Self-efficacy mechanism in human agency". American Psychologist, 1982, 37, 122-147.

BERNSTEIN, D. A., y NerTZEL, M. T.: «Demand characteristics in behavior modification: The natural history of a "nuisance"", en M. Hernsen, R. M. Eisler y P. M. Miller (eds.): Progress in bebavior modification (vol. 4). Nueva York: Academic Press, 1977.

BoOTZIN, R. B., y LICK, J. R.: «Expectancies in therapy research: Interpretive artifact or mediating mechanism?» Jourmal of Consulting and Clinical Psychology, 1979, 47, 852-855.

BYERLY, H.: «Explaining and exploiting the placebo effect». Perspectives in Biology and Medicine, 1976, 3, 423-435.

EYSENCK, H. J.: «The effects of psychotherapy», en H. J. Eysenck (ed.): Handbook of abnormal psychology: An experimental approach. Nueva York: Basic Books, 1961.

FRANK, J. D.: Persuasion and healing. Baltimore, MD: John Hopkins University Press, 1961.

GARFIELD, S. C.: «Psychorherapy: A 40-year appraisal». American Psychologist, 1981, 35, 174-183.

JACOBSON, N. S., y BAUCOM, D. H.: «Design and assessment of nonspecific control groups in behavior modification research». Bebavior Therapy, 1977, 8, 709-719.

KAZDIN, A. E.: «Nonspecific treatment factors in psychotherapy outcome research». Journal of Consulting and Clinical Psychology, 1979a, 47, 846-851.

KAzDIN, A. E.: «Therapy outcome questions requiring control of credibility and treatment-generated expectancies». Behavior Therapy, 1979b, 10, 81-93.

KAzDIN, A. E. y WILCOXON, L. A.: «Systematic desensitization and nonspecific treatment effects: A methodological evaluation». Psychological Bulletin, 1976, 83, 729-758.

KIRSCH, I.: «The placebo effect and the cognitive-behavioral revolution». Cognitive Therapy and Research, 1978, $2,255-264$.

LICK, J.: «Expectancy, false galvanic skin response feedback, and systematic desensitization in the modification of phobic behaviorm. Journal of Consulting and Clinical Psychology, 1975, 43, 557-567.

LUBORSKY, L.; Singer, B., y LUBORSKY, L.: «Comparative studies of psychotherapy». Archives of General Psychiatry, 1975, 32, 995-1008.

MAHONEY, M. J.: «Cognitive therapy and research: A question of questions». Cognitive Therapy and Research, $1977,1,5-16$. 
McReynolds, W. T.; BARnes, A. R.; Brooks, S., y Rehagen, N. J.: \&The role of attention-placebo influences in the efficacy of systematic desensitization». Journal of Consulting and Clinical Psychology, 1973, 41, 86-92.

Neumann, K. F.; CruTell, J. W., y SCHneIDER, L.: The stress inoculation treatment of male beterosocial anxiety: An outcome study. Trabajo presentado al encuentro de la American Psychologiçal Association, Los Angeles, agosto de 1981 .

O'LeARY, K. D., y BORKovec, T. D.: «Conceptual methodological, and ethical problems 'of placebo groups in psychotherapy research". American Psychologist, 1978, 33, 821-830.

PAUL, G. L.: Insight vs. desensitization in psychotherapy. Stanford, CA: Stanford University Press, 1966.

Rosenthal, D., y Frank, J. D.: «Psychotherapy and the placebo effect». Psychological Bulletin, 1956, 53, $294-302$.

ShaPIRo, A. K.: «Placebo effects in medicine psychotherapy, and psychoanalysis», en $\lambda$ A. E. Bergin y S. C. Garfield (eds.), Handbook of psychotberapy and behavior change: Empirical analysis. Nueva York: Wiley. 1971.

Shapiro, A. K., y Morris, L. A.: «The placebo effect in medical and psychological therapies», en S. C. Garfield y A. E. Bergin (eds.), Handbook of psychotherapy and bebavior change. Nueva York: Wiley, 1978.

SKINNER, B. F.: About bebaviorism. Nueva York: Knopf, 1974.

SMITH, M. L., y GLASS, G. V.: «Meta-analysis of psychotherapy outcome studies». American Psychologist, 1977, $32,752-760$.

TORI, C., y WORRELI, L.: «Reduction of human avoidant behavior: A comparison of counterconditioning, expectancy, and cognitive information approaches». Journal of Consulting and Clinical Psychology, 1973, 41, 269-278.

WILKINS, W.: «Expectancies in therapy research: Discriminating among heterogeneous nonspecifics». Journal of Consulting and Clinical Psycbology, 1979a, 47, 837-845.

WILKINS, W.: «Heterogeneous referents, indiscriminate language, and complementary research purposes». Journal of Consulting and Clinical Psychology, 1979b, 47, 856-859.

WILKINS, W.: «Failure of placebo groups to control for nonspecific events in therapy outcome research». Psychotherapy: Theory, Research, and Practice, 1983, 20, 31-40. 